\title{
Research Article \\ Continuous Time Portfolio Selection under Conditional Capital at Risk
}

\section{Gordana Dmitrasinovic-Vidovic, Ali Lari-Lavassani, Xun Li, and Antony Ware}

The Mathematical and Computational Finance Laboratory, University of Calgary, Calgary, $A B$, Canada T2N $1 N 4$

Correspondence should be addressed to Gordana Dmitrasinovic-Vidovic, gdmitras@ucalgary.ca

Received 29 October 2009; Revised 4 February 2010; Accepted 26 March 2010

Academic Editor: Ričardas Zitikis

Copyright (C) 2010 Gordana Dmitrasinovic-Vidovic et al. This is an open access article distributed under the Creative Commons Attribution License, which permits unrestricted use, distribution, and reproduction in any medium, provided the original work is properly cited.

Portfolio optimization with respect to different risk measures is of interest to both practitioners and academics. For there to be a well-defined optimal portfolio, it is important that the risk measure be coherent and quasiconvex with respect to the proportion invested in risky assets. In this paper we investigate one such measure - conditional capital at risk - and find the optimal strategies under this measure, in the Black-Scholes continuous time setting, with time dependent coefficients.

\section{Introduction}

The choice of risk measure has a significant effect on portfolio investment decisions. Downside risk measures-that focus attention on the downside tail of the distribution of portfolio returns-have received considerable attention in the financial world. Value at risk (VaR) is probably the most famous among these measures, having featured heavily in various regulatory frameworks. It can be defined for a random variable $X$ and a confidence level $\alpha$ by $\operatorname{VaR}(X)=E[X]-q_{\alpha}$, where $q_{\alpha}$ is the $\alpha$-quantile of $X$ (see e.g., [1, Equation (1.2)]) (Another common definition is that the VaR of a loss distribution $L$ is the smallest number $x_{\alpha}$ such that $P\left[L>x_{\alpha}\right]=\alpha$. This is equivalent to the definition given here if we define the loss of the portfolio $X$ to be given by $L=E[X]-X$, and identify $x_{\alpha}=\operatorname{VaR}(X)$.). A closely-related downside risk measure is capital at risk (CaR), defined in [2] (see also $[3,4])$ as the difference between the riskless investment and the quantile $q_{\alpha}$.

Quantile-based risk measures such as VaR and CaR suffer from several shortcomings. First, while they measure the best of the worst outcomes at the $100(1-\alpha) \%$ confidence level, they do not answer the question of how severe the loss can be. Also, one of the 
most important concerns is that these measures are not in general subadditive; that is, when used to measure risk, they do not always satisfy the notion that the diversification should not create more risk [5]. Finally, as illustrated in [6], VaR can exhibit multiple local extrema.

These issues were addressed in the widely cited article by Artzner at al. [7], where the authors define coherent risk measures by four conditions that such measures should satisfy. The article motivated a number of authors $[5,8-13]$ to propose and investigate different types of coherent risk measures, all of which are tail mean-based risk measures.

One such measure, that does not suffer from the critical shortcomings of VaR and CaR, is conditional capital at risk (CCaR). This is defined (in [14]) as the difference between the riskless investment and the conditional expected wealth, under the condition that the wealth is smaller than the corresponding quantile, for a given risk level. As such, this measure provides an indication of the likely severity of the loss in the event that the loss exceeds a given quantile. In this paper we prove that $\mathrm{CCaR}$ is strongly quasiconvex as a function of the portfolio, which is an essential property for optimization. We investigate conditional capital at risk in a multiasset Black-Scholes setting, in continuous time, and with time-dependent coefficients. We generalize and extend the optimization approach of Emmer at al. (see $[2,14])$ to the continuous-time setting.

The outline of this paper is as follows. In Section 2, we give the notation and define the portfolio process and $\mathrm{CCaR}$. Section 3 provides the proof that $\mathrm{CCaR}$ is a coherent risk measure and that it satisfies the property of strong quasiconvexity. In Section 4 , we derive an analytical solution for the minimal CCaR problem, up to a scalar constant which has to be evaluated numerically. Section 5 is devoted to the derivation of an analytical strategy for the maximal expected wealth, subject to constrained CCaR. Section 6 provides some numerical examples, and Section 7 concludes this paper.

\section{Preliminaries}

We introduce the following notation. The $m$-dimensional column vector with each component equal to 1 is denoted by $e$, the Euclidean norm of a matrix or vector by $\|\cdot\|$, and the space of $\mathbb{R}^{n}$-valued, square-integrable functions defined on $[0, t]$ by $L^{2}\left([0, t], \mathbb{R}^{n}\right)$, or just $L^{2}$. The natural inner product of this space is denoted by $\langle\cdot, \cdot\rangle_{t}$, and the corresponding norm by $\|\cdot\|_{t}$.

We work under the following assumptions.

Assumption 2.1. (i) The securities are perfectly divisible.

(ii) Negative positions in securities are possible.

(iii) Rebalancing of the holdings does not lead to transaction costs.

Assumption 2.2. (i) $m+1$ assets are traded continuously over a finite horizon $[0, T]$.

(ii) $m$ of these assets are stocks that follow the generalized Black-Scholes dynamics:

$$
d S_{i}(t)=S_{i}(t)\left(b_{i}(t) d t+\sum_{j=1}^{m} \sigma_{i j}(t) d W^{j}(\mathrm{t})\right), \quad t \in[0, T], S_{i}(0)>0, i=1, \ldots, m
$$

where $W^{j}(t), j=1, \ldots, m$, are independent standard Brownian motions. 
(iii) One of the assets is a bond, whose price $S_{0}(t), t \geq 0$, evolves according to the differential equation:

$$
d S_{0}(t)=r(t) S_{0}(t) d t, \quad t \in[0, T], S_{0}(0)=S_{0}>0,
$$

where $r(t)(>0)$ is the interest rate of the bond. Throughout this work, we assume that borrowing in the bond is unconstrained.

(iv) The volatility matrix $\sigma(t)$ (with ijth element $\sigma_{i j}(t)$ ), its inverse $\sigma^{-1}(t)$, the drift vector $b(t):=\left(b_{1}(t), \ldots, b_{m}(t)\right)^{\prime}$, and the interest rate $r(t)$ are deterministic, Borel measurable, bounded functions over $[0, T]$, so that they belong to the appropriate $L^{2}$ spaces.

(v) $\sigma(t)$ satisfies the nondegeneracy condition:

$$
x^{\prime} \sigma(t) \sigma(t)^{\prime} x \geq \delta x^{\prime} x, \quad \forall t \in[0, T], \forall x \in \mathbb{R}^{m},
$$

where $\delta>0$ is a given constant.

We note that, under the above assumptions, the market is complete.

At any time $t, N_{i}(t)$ shares are held in the asset $S_{i}(t)$, leading to the wealth $X^{\pi}(t)=$ $\sum N_{i}(t) S_{i}(t)$. The $m+1$-dimensional vector-valued function $N(t)=\left(N_{0}(t), \ldots, N_{m}(t)\right)^{\prime}$ is called the trading strategy. We denote the fraction of the wealth $X^{\pi}(t)$ invested into the risky asset $S_{i}(t)$ by

$$
\pi_{i}(t)=\frac{N_{i}(t) S_{i}(t)}{X^{\pi}(t)}, \quad i=1, \ldots, m,
$$

and call $\pi(t):=\left(\pi_{1}(t), \ldots, \pi_{m}(t)\right)^{\prime} \in \mathbb{R}^{m}$ the portfolio. The fraction held in the bond is $\pi_{0}(t)=$ $1-\pi^{\prime}(t) e$. Under the assumption that the trading strategy is self-financing, the wealth process follows the dynamics

$$
d X^{\pi}(t)=X^{\pi}(t)\left(\left(r(t)+B(t)^{\prime} \pi(t)\right) d t+\pi^{\prime}(t) \sigma(t) d W(t)\right), \quad X(0)=X_{0}
$$

where $X_{0}$ is the initial wealth, and the risk premium vector $B(t)$ is defined by

$$
B(t):=b(t)-r(t) e, \quad t \in[0, T]
$$

Proceeding as in [3], to ensure a minimal tractability of the optimization problems which we solve through the following sections, we restrict our attention in this work to the class $Q$ of portfolios $\pi(\cdot)$ which are Borel measurable, deterministic, and bounded over $[0, T]$. Such portfolios are called admissible. Note that for an admissible portfolio $\pi(\cdot),(2.5)$ is guaranteed to have a strong solution $X^{\pi}(\cdot)$ (see [15, Theorem 5.2.9]). Note that, by allowing for time-dependent coefficients, this generalises the class of portfolios considered in [14].

Under condition (2.3) the market price of risk is uniquely defined by

$$
\theta(t)=\sigma(t)^{-1} B(t)
$$


It will be shown throughout this work that the magnitude of the $L^{2}$-norm of the market price of risk is the determining criterion for optimal investment strategies, which turn out to be the weighted averages of the bond and Merton's portfolio defined by

$$
\pi_{M}(t):=(\sigma(t) \sigma(t))^{-1} B(t)
$$

This result is an illustration of the mutual fund theorem in complete markets.

We now recall the definitions of the quantiles and quantile-based risk measures for $X^{\pi}(t)$.

Definition 2.3. Suppose that $F_{t}(x)$ is the cumulative distribution function of the wealth $X^{\pi}(t)$, at time $t \in[0, T]$. For a risk level $\alpha \in(0,0.5)$, the $\alpha$-quantile of $X^{\pi}(t)$ is defined as

$$
q_{\alpha}\left(X_{0}, \pi, t\right):=\inf \{x \in \mathbb{R} \mid F(x) \geq \alpha\} .
$$

The tail mean or expected shortfall of the wealth process $X^{\pi}(t)$, which we denote by $\operatorname{TM}_{\alpha}\left(X^{\pi}(t)\right)$, is the expected value of $X^{\pi}(t)$ conditional on $X^{\pi}(t) \leq q_{\alpha}\left(X_{0}, \pi, t\right)$, that is,

$$
\mathrm{TM}_{\alpha}\left(X^{\pi}(t)\right):=E\left[X^{\pi}(t) \mid X^{\pi}(t) \leq q_{\alpha}\left(X_{0}, \pi, t\right)\right]
$$

The conditional capital at risk, which we denote by $\operatorname{CCaR}\left(X_{0}, \pi, t\right)$, is defined to be the difference between the riskless investment and the tail mean, that is,

$$
\operatorname{CCaR}\left(X_{0}, \pi, t\right):=X_{0} R(t)-\mathrm{TM}_{\alpha}\left(X^{\pi}(t)\right),
$$

where $R(t)$ is defined as

$$
R(t)=\exp \left(\int_{0}^{t} r(s) d s\right) .
$$

Remark 2.4. Note that, with CCaR defined in this way, we get the following.

(i) An investment in a riskless asset corresponds to zero CCaR.

(ii) An increase in the tail mean corresponds to a decrease in CCaR. Thus, negative $\mathrm{CCaR}$ corresponds to the case when the tail mean is above the riskless return, that is, a desired result, while positive CCaR corresponds to the case when the tail mean is below the riskless return which we try to reverse by minimizing CCaR.

It was shown in [3] that the $\alpha$-quantile of the wealth process $X^{\pi}(t)$ can be written as

$$
q_{\alpha}\left(X_{0}, \pi, t\right)=X_{0} R(t) \exp \left(\langle B, \pi\rangle_{t}-\frac{1}{2}\left\|\sigma^{\prime} \pi\right\|_{t}^{2}-\left|z_{\alpha}\right|\left\|\sigma^{\prime} \pi\right\|_{t}\right)
$$


where, for a given risk level $\alpha, z_{\alpha}$ denotes the corresponding $\alpha$-quantile of the standard normal distribution (note that this is negative when $\alpha<0.5$ ). In the following, we let $\varphi$ and $\Phi$ denote the density and cumulative distribution functions of the standard normal random variable. We have the following proposition.

Proposition 2.5. The tail mean of the wealth process $X^{\pi}(t)$ solving (2.5) can be expressed as

$$
T M_{\alpha}\left(X^{\pi}(t)\right)=X_{0} R(t) \frac{1}{\alpha} \exp \left(\langle B, \pi\rangle_{t}\right) \Phi\left(-\left|z_{\alpha}\right|-\left\|\sigma^{\prime} \pi\right\|_{t}\right),
$$

and the expected value is given by

$$
E\left[X^{\pi}(t)\right]=X_{0} R(t) \exp \left(\langle B, \pi\rangle_{t}\right)
$$
corollary.

The proof is given in the appendix. From Proposition 2.5 we get the following

Corollary 2.6. The conditional capital at risk of the solution to (2.5) can be written as

$$
\operatorname{CCaR}\left(X_{0}, \pi, t\right)=X_{0} R(t)\left(1-\frac{1}{\alpha} \exp \left(\langle B, \pi\rangle_{t}\right) \Phi\left(-\left|z_{\alpha}\right|-\left\|\sigma^{\prime} \pi\right\|_{t}\right)\right) .
$$

Throughout the paper we will use the following function:

$$
g_{\alpha}(\pi, t):=\langle B, \pi\rangle_{t}+\ln \left(\Phi\left(-\left|z_{\alpha}\right|-\left\|\sigma^{\prime} \pi\right\|_{t}\right)\right)-\ln \alpha,
$$

which transforms the expression for conditional capital at risk into

$$
\operatorname{CCaR}\left(X_{0}, \pi, t\right)=X_{0} R(t)\left(1-\exp \left(g_{\alpha}(\pi, t)\right)\right) .
$$

We now turn our attention to investigating the properties of CCaR.

\section{Coherency and Quasiconvexity of CCaR}

The notion of coherent risk measures has been widely discussed in the recent literature (see [5, 7-13] e. g.). Below we recall the definition of coherency, confirm that CCaR is a coherent risk measure, and prove that it is quasiconvex.

Definition 3.1. Let $V$ be the set of real-valued random processes on a complete probability space $(\Omega, \mathcal{F}, P)$, with its natural filtration $\left\{\mathcal{F}_{t}\right\}_{t \in[0, T]}$, satisfying $E[S(t)]<\infty$ for all $S(t) \in V$, and for $t \in[0, T]$. Then $\rho: V \rightarrow \mathbb{R}$ is a coherent risk measure if it satisfies the following properties. 
(1) Subadditivity: $\rho$ is subadditive if, for all random processes $S(t), Y(t) \in V$,

$$
\rho(S(t)+Y(t)) \leq \rho(S(t))+\rho(Y(t)) \text { for } t \in[0, T]
$$

(2) Positive Homogeneity: $\rho$ is positive homogeneous if, for all $S(t) \in V$ and constant $c>0$,

$$
\rho(c S(t))=c \rho(S(t)) \quad \text { for } t \in[0, T]
$$

(3) Monotonicity: $\rho$ is monotone if, for all $S(t), Y(t) \in V$, such that $S(t) \geq Y(t)$ almost everywhere, and $S_{0}=Y_{0}$,

$$
\rho(S(t)) \leq \rho(Y(t)) \quad \text { for } t \in[0, T] .
$$

(4) Translation Invariance: $\rho$ is translation invariant if, for all $S(t) \in V$ and $c \in \mathbb{R}$,

$$
\rho(S(t)+c)=\rho(S(t)) \quad \text { for } t \in[0, T]
$$

To prove that $\mathrm{CCaR}\left(X_{0}, \pi, t\right)$ of a wealth process $X^{\pi}(t) \in V$ is a coherent risk measure we refer to [8, Definition 2.6], where the expected shortfall of a random process $X(t)$, with the corresponding $\alpha$-quantile $q_{\alpha}$, is defined as

$$
\mathrm{ES}_{\alpha}(X(t))=-\alpha^{-1}\left(E\left[X(t) I_{X(t) \leq q_{x}}\right]+q_{x}\left(\alpha-P\left(X(t) \leq q_{x}\right)\right)\right)
$$

and is shown to be coherent [8, Proposition 3.1]. We should note that the definition of coherency used in [8] involves translation invariance in the sense that $\rho(X(t)+c)=\rho(X(t))-c$, for all $X(t) \in V$ and $c \in \mathbb{R}$.

In order to relate the result in [8] to the coherency of CCaR in the sense used here, we first note that, if $X(t)$ is a random process with a continuous probability distribution, we have $\mathrm{ES}_{\alpha}(X(t))=-\mathrm{TM}_{\alpha}(X(t))$, so that (with a slight abuse of notation) $\mathrm{CCaR}(X(t))=$ $X_{0} R(t)+\mathrm{ES}_{\alpha}(X(t))$. If we consider the shifting of the portfolio value by an amount $c$, we have

$$
\begin{aligned}
\operatorname{CCaR}(X(t)+c) & =X_{0} R(t)+c+\mathrm{ES}_{\alpha}(X(t)+c) \\
& =X_{0} R(t)+c+\mathrm{ES}_{\alpha}(X(t))-c \\
& =X_{0} R(t)+\mathrm{ES}_{\alpha}(X(t))=\operatorname{CCaR}(X(t)),
\end{aligned}
$$

and so we have the following result.

Corollary 3.2. Conditional capital at risk $\operatorname{CCaR}\left(X_{0}, \pi, t\right)$ of a wealth process $X^{\pi}(t) \in V$, at time $t \in[0, T]$, for a risk level $\alpha \in(0,0.5)$, is a coherent risk measure. 
Remark 3.3. (i) While properties 2 and 3 are quite natural, subadditivity has the obvious, yet important consequence that diversification does not create more risk.

(ii) Properties 1 and 2 together are very important as they guarantee the convexity of the risk measure which is essential for optimization.

(iii) In our definition of a coherent risk measure we say that a risk measure is translation invariant, if $\rho(X(t)+c)=\rho(X(t))$, for all $X(t) \in V$, and $c \in \mathbb{R}$. While in article [8], It is said that arisk measure is translation invariant, if $\rho(X(t)+c)=\rho(X(t))-c$, for all $X(t) \in V$ and $c \in \mathbb{R}$ It is our belief that the definition of the translation invariance, as given in this paper, corresponds more with the intuition behind the notion of translation invariance than the definition given in [8].

Remark 3.4. In the above remarks we investigated the impact of diversification to the portfolio risk, as measured by CCaR. We saw that the axiom of subadditivity requires that the risk of the sum of two risky processes, say two stock price processes, be less than or equal to the sum of the individual risks associated with these stock price processes. This means that diversification does not create more risk, as measured by $\mathrm{CCaR}$, and that, as long as we diversify, we expect risk reduction.

However, if the portfolio consists of all market assets, the diversification is completed. Then the risk can be further reduced by optimization, that is, by rebalancing the positions across these assets. We then look at the risk measure $\mathrm{CCaR}$ as a function of the portfolio $\pi$, and prove that it is strongly quasiconvex in $\pi$ which further implies the uniqueness of the corresponding optimization problems' solutions.

We now turn to the notion of strong quasiconvexity, which we note has not been discussed in the context of portfolio optimization in any of the quoted references except in $[3,4]$. Its usefulness lies in its role in establishing the existence of unique solutions to portfolio optimization problems.

We first recall (see [16, Definition 3.5.8]) that a function $f: U \subset \mathbb{R}^{m} \rightarrow \mathbb{R}$ is said to be strongly quasiconvex if

$$
f(\lambda \pi+(1-\lambda) \xi)<\max \{f(\pi), f(\xi)\} \quad \forall \pi, \xi \in U, \pi \neq \xi, \lambda \in(0,1) .
$$

In the following theorem we prove that $\mathrm{CCaR}$ has this important property, when viewed as a function of the portfolio weights.

Theorem 3.5. For all distinct $\pi(\cdot), \xi(\cdot) \in \mathcal{Q}$ (where the set $\{t \in[0, T] \mid \pi(t) \neq \xi(t)\}$ has a positive Lebesgue measure), and for all $\lambda \in(0,1)$

$$
\operatorname{CCaR}\left(\mathrm{X}_{0}, \lambda \pi+(1-\lambda) \xi, T\right)<\max \left\{\operatorname{CCaR}\left(\mathrm{X}_{0}, \pi, T\right), \operatorname{CCaR}\left(\mathrm{X}_{0}, \xi, T\right)\right\} .
$$

Proof of Theorem 3.5. We suppose, without loss of generality, that

$$
\operatorname{CCaR}\left(\mathrm{X}_{0}, \pi, T\right)>\operatorname{CCaR}\left(\mathrm{X}_{0}, \xi, T\right),
$$

in which case, from (2.18), we have

$$
g_{\alpha}(\pi, T)<g_{\alpha}(\xi, T)
$$


Let $\lambda \in(0,1)$. We claim that

$$
\operatorname{CCaR}\left(X_{0}, \lambda \pi+(1-\lambda) \xi, T\right)<\operatorname{CCaR}\left(X_{0}, \pi, T\right),
$$

which is equivalent to the statement

$$
g_{\alpha}(\lambda \pi+(1-\lambda) \xi, T)>g_{\alpha}(\pi, T)
$$

For ease of notation, we define the function

$$
\gamma(x):=\ln \left(\Phi\left(-\left|z_{\alpha}\right|-x\right)\right)
$$

Since $g_{\alpha}(\pi, T)=\langle B, \pi\rangle_{T}-\ln \alpha+\gamma\left(\left\|\sigma^{\prime} \pi\right\|_{T}\right),(3.10)$ is equivalent to

$$
\langle B, \xi-\pi\rangle_{T}+\gamma\left(\left\|\sigma^{\prime} \xi\right\|_{T}\right)-\gamma\left(\left\|\sigma^{\prime} \pi\right\|_{T}\right)>0,
$$

while (3.12) becomes

$$
(1-\lambda)\langle B, \xi-\pi\rangle_{T}+\gamma\left(\left\|\sigma^{\prime}(\lambda \pi+(1-\lambda) \xi)\right\|_{T}\right)-\gamma\left(\left\|\sigma^{\prime} \pi\right\|_{T}\right)>0
$$

Clearly, from (3.14), the left-hand side of (3.15) is greater than

$$
\gamma\left(\left\|\sigma^{\prime}(\lambda \pi+(1-\lambda) \xi)\right\|_{T}\right)-\lambda \gamma\left(\left\|\sigma^{\prime} \pi\right\|_{T}\right)-(1-\lambda) \gamma\left(\left\|\sigma^{\prime} \xi\right\|_{T}\right),
$$

and the theorem will be proved if we can establish that this is nonnegative. In order to do this, we make use of the following lemma, the proof of which is given in the appendix.

Lemma 3.6. The function $k(x)=\ln \Phi(-x)$ is decreasing and strictly concave for all $x>0$.

Since $\gamma(x)=k\left(\left|z_{\alpha}\right|+|x|\right)$, one can make use of Lemma 3.6 to establish the following:

$$
\begin{aligned}
r\left(\left\|\sigma^{\prime}(\lambda \pi+(1-\lambda) \xi)\right\|_{T}\right) & \geq \gamma\left(\lambda\left\|\sigma^{\prime} \pi\right\|_{T}+(1-\lambda)\left\|\sigma^{\prime} \xi\right\|_{T}\right) \\
& \geq \lambda \gamma\left(\left\|\sigma^{\prime} \pi\right\|_{T}\right)+(1-\lambda) \gamma\left(\left\|\sigma^{\prime} \xi\right\|_{T}\right)
\end{aligned}
$$

The proof is complete.

This theorem has an immediate, important consequence. Namely, from [16, Theorem 3.5.9], if a function $f: U \subset \mathbb{R}^{m} \rightarrow \mathbb{R}$ is strongly quasiconvex, then its local minimum is its unique global minimum. Therefore, the following corollary is true.

Corollary 3.7. If $\operatorname{CCaR}\left(X_{0}, \pi, T\right)$ has a local minimum at $\pi^{*}(\cdot) \in Q$, then $\pi^{*}(\cdot)$ is its unique global minimum.

This guarantees that minimization of $\operatorname{CCaR}\left(X_{0}, \pi, T\right)$ with respect to $\pi$ gives the true, global minimum. To illustrate strong quasiconvexity, we give the plot of $\mathrm{CCaR}$ for 


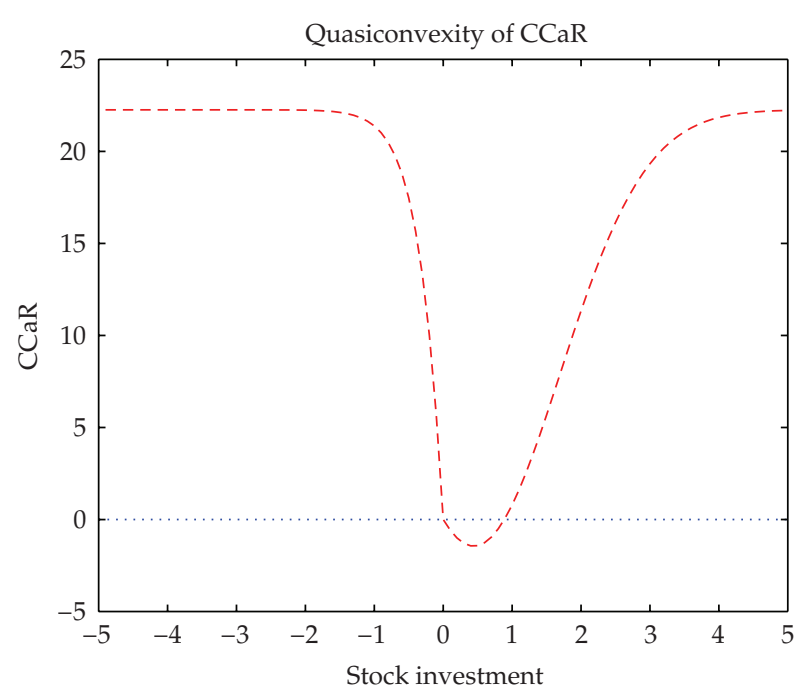

Figure 1: The graph of CCaR as a function of the fraction of wealth $\pi$ invested in the stock, for $\pi \in[-5,5]$, $T=16, S(0)=10, r=0.05, \sigma=0.2, b=0.15$. Observe that, in this case (with a long-time horizon and constantly high-performing stocks), the global minimum $\pi_{\varepsilon}$ lies in the interval $[0,1]$ and satisfies $\operatorname{CCaR}\left(\pi_{\varepsilon}\right)<0$.

the following example (where the parameters were chosen to represent rather extreme conditions, for the purposes of illustration).

Example 3.8. We consider a market consisting of one stock following the SDE

$$
d S(t)=S(t)(0.15 d t+0.2 d W(t)), \quad t \in[0,16], S(0)=10,
$$

and the bond with the constant interest rate $r=0.05$. The graph of $\operatorname{CCaR}(\pi)$ is given in Figure 1.

\section{Minimal Conditional Capital at Risk}

The first portfolio selection problem we consider is to minimize risk as measured by CCaR, that is, to determine its minimal value over all $\pi \in \mathcal{Q}$. The problem can be stated as

$$
\min _{\pi \in Q} \operatorname{CCaR}\left(X_{0}, \pi, T\right) .
$$

Using (2.18), problem (4.1) transforms into

$$
\min _{\pi \in Q} X_{0} R(T)\left(1-\exp \left(g_{\alpha}(\pi, T)\right)\right)
$$

Since $R(T)$ is a constant, problem (4.2) is equivalent to

$$
\max _{\pi \in Q} g_{\alpha}(\pi, T) .
$$


We now introduce the fundamental dimension reduction procedure, used throughout this work. Following $[2,14]$, we project the optimization problems considered in this paper onto the family of surfaces $Q_{\varepsilon}=\left\{\pi(\cdot) \in L^{2}:\left\|\sigma^{\prime} \pi\right\|_{T}^{2}=\varepsilon^{2}\right\}$, and note that $Q=\bigcup_{\varepsilon \geq 0} Q_{\varepsilon}$.

We denote by $g_{\alpha}^{\varepsilon}$ the restriction of $g_{\alpha}$ to $Q_{\varepsilon}$, so that

$$
g_{\alpha}^{\varepsilon}(\pi, T)=\langle B, \pi\rangle_{T}+\ln \left(\Phi\left(-\left|z_{\alpha}\right|-\varepsilon\right)\right)-\ln \alpha .
$$

Taking into account the definition of $Q_{\varepsilon}$, problem (4.3) can be stated as

$$
\max _{\varepsilon \geq 0} \max _{\pi \in Q_{\varepsilon}} g_{\alpha}^{\varepsilon}(\pi, T)
$$

We deal with this problem in two stages. First, fixing $\varepsilon$ reduces the problem to

$$
\max _{\pi \in Q_{\varepsilon}} g_{\alpha}^{\varepsilon}(\pi, T)
$$

If $\pi_{\varepsilon}$ denotes the unique maximising portfolio for this problem, then (4.5) can be solved through the one-dimensional problem:

$$
\max _{\varepsilon \geq 0} g_{\alpha}^{\varepsilon}\left(\pi_{\varepsilon}, T\right)
$$

It remains to solve the subproblem (4.6). Since $\varepsilon$ is fixed, we see from (4.4) that (4.6) is equivalent to the problem

$$
\max _{\pi \in Q_{\varepsilon}}\langle B, \pi\rangle_{T}
$$

the solution of which is given by the following proposition (the proof of which is given in [6, Proposition 2.1]).

Proposition 4.1. For a fixed $\varepsilon$, the solution of problem (4.6) is attained by

$$
\pi_{\varepsilon}(t)=\frac{\varepsilon}{\|\theta\|_{T}}\left(\sigma(t) \sigma(t)^{\prime}\right)^{-1} B(t)
$$

where $\theta$ denotes the market price of risk, defined in (2.7).

We are now ready to solve problem (4.3), and we have the following theorem.

Theorem 4.2. Let $\theta(\cdot)$ be the market price of risk $(2.7) \cdot g_{\alpha}(\pi, T)$ attains its maximum when

$$
\pi(t):=\pi_{\varepsilon^{*}}(t)=\varepsilon^{*}\|\theta\|_{T}^{-1}\left(\sigma(t) \sigma(t)^{\prime}\right)^{-1} B(t),
$$

where, if $\|\theta\|_{T} \geq \varphi\left(\left|z_{\alpha}\right|\right) / \alpha, \varepsilon^{*}$ is defined to be the unique solution of the equation

$$
\frac{\varphi\left(\left|z_{\alpha}\right|+\varepsilon\right)}{\Phi\left(-\left|z_{\alpha}\right|-\varepsilon\right)}=\|\theta\|_{T}
$$


and, if $\|\theta\|_{T}<\varphi\left(\left|z_{\alpha}\right|\right) / \alpha, \varepsilon^{*}=0$. The corresponding minimum conditional capital at risk is

$$
\operatorname{CCaR}\left(X_{0}, \pi_{\varepsilon^{*}}, T\right)=X_{0} R(T)\left(1-\frac{1}{\alpha} \exp \left(\varepsilon^{*}\|\theta\|_{T}\right) \Phi\left(-\left|z_{\alpha}\right|-\varepsilon^{*}\right)\right)
$$

(which is 0 if $\varepsilon^{*}=0$ ), and the expected wealth is

$$
E\left[X^{\pi_{\varepsilon^{*}}}(T)\right]=X_{0} R(T) \exp \left(\varepsilon^{*}\|\theta\|_{T}\right) .
$$

Proof of Theorem 4.2. Using the definition of $\theta(t)$ and substituting (4.9) into (4.5) allows us to rewrite (4.5) as

$$
\max _{\varepsilon \geq 0} g_{\alpha}^{\varepsilon}\left(\pi_{\varepsilon}, T\right):=\varepsilon\|\theta\|_{T}+\ln \left(\Phi\left(-\left|z_{\alpha}\right|-\varepsilon\right)\right)-\ln \alpha
$$

If we define the function

$$
f(\varepsilon):=g_{\alpha}^{\varepsilon}\left(\pi_{\varepsilon}, T\right)=\varepsilon\|\theta\|_{T}+\ln \left(\Phi\left(-\left|z_{\alpha}\right|-\varepsilon\right)\right)-\ln \alpha,
$$

we get

$$
\begin{aligned}
& f^{\prime}(\varepsilon)=\|\theta\|_{T}-\frac{\varphi\left(-\left|z_{\alpha}\right|-\varepsilon\right)}{\Phi\left(-\left|z_{\alpha}\right|-\varepsilon\right)}=\|\theta\|_{T}-\frac{\varphi\left(\left|z_{\alpha}\right|+\varepsilon\right)}{\Phi\left(-\left|z_{\alpha}\right|-\varepsilon\right)}, \\
& f^{\prime}(0)=\|\theta\|_{T}-\frac{\varphi\left(\left|z_{\alpha}\right|\right)}{\Phi\left(-\left|z_{\alpha}\right|\right)}=\|\theta\|_{T}-\frac{\varphi\left(\left|z_{\alpha}\right|\right)}{\alpha}, \\
& f^{\prime \prime}(\varepsilon)=\frac{\varphi\left(\left|z_{\alpha}\right|+\varepsilon\right)}{\left(\Phi\left(-\left|z_{\alpha}\right|-\varepsilon\right)\right)^{2}}\left(\left(1-\Phi\left(\left|z_{\alpha}\right|+\varepsilon\right)\right)\left(\left|z_{\alpha}\right|+\varepsilon\right)-\varphi\left(\left|z_{\alpha}\right|+\varepsilon\right)\right) .
\end{aligned}
$$

We see that $f^{\prime \prime}(\varepsilon)$ has the same form as $k^{\prime \prime}(x)$, where $k(x)$ is defined in Lemma 3.6, with $\left|z_{\alpha}\right|+\varepsilon$, instead of $x$, so that $f^{\prime \prime}(\varepsilon) \leq 0$. Since $f^{\prime}(\varepsilon)$ is a decreasing function of $\varepsilon$ for $\varepsilon>0$, we have two cases.

(i) If $f^{\prime}(0) \geq 0$, that is, $\|\theta\|_{T} \geq \varphi\left(\left|z_{\alpha}\right|\right) / \alpha$, then the equation

$$
f^{\prime}(\varepsilon)=\|\theta\|_{T}-\frac{\varphi\left(\left|z_{\alpha}\right|+\varepsilon\right)}{\Phi\left(-\left|z_{\alpha}\right|-\varepsilon\right)}=0
$$

has a unique solution which we denote by $\varepsilon^{*}$. Since $f(\varepsilon)$ is a concave function, it reaches its maximum at $\varepsilon^{*}$.

(ii) If $f^{\prime}(0)<0$, that is, $\|\theta\|_{T}<\varphi\left(\left|z_{\alpha}\right|\right) / \alpha$, then $f^{\prime}(\varepsilon)<0$ for all $\varepsilon \geq 0$, and so (4.17) has no solution. This implies that $f(\varepsilon)$ is decreasing for all $\varepsilon \geq 0$, so that the optimal solution to problem (4.14) is $\varepsilon=0$. 
To complete the proof of Theorem 4.2, note that (4.9), with $\varepsilon=\varepsilon^{*}$, is the optimal solution of problem (4.3). One can then write

$$
\left\langle B, \pi_{\varepsilon^{*}}\right\rangle_{T}=\frac{\varepsilon^{*}}{\|\theta\|_{T}}\left\|\sigma^{-1} B\right\|_{T}^{2}=\varepsilon^{*}\|\theta\|_{T}, \quad\left\|\sigma^{\prime} \pi\right\|_{t}^{2}=\varepsilon^{* 2},
$$

leading to

$$
\begin{gathered}
\operatorname{CCaR}\left(X_{0}, \pi_{\varepsilon^{*}}, T\right)=X_{0} R(T)\left(1-\frac{1}{\alpha} \exp \left(\varepsilon^{*}\|\theta\|_{T}\right) \Phi\left(-\left|z_{\alpha}\right|-\varepsilon^{*}\right)\right), \\
E\left[X^{\pi_{\varepsilon^{*}}}(t)\right]=X_{0} R(T) \exp \left(\varepsilon^{*}\|\theta\|_{T}\right) .
\end{gathered}
$$

Remark 4.3. (i) Note that, if $\|\theta\|_{T} \geq \varphi\left(\left|z_{\alpha}\right|\right) / \alpha$, we can deduce by using (A.12) that

$$
\|\theta\|_{T} \geq \frac{\varphi\left(\left|z_{\alpha}\right|\right)}{\alpha}=\frac{\varphi\left(\left|z_{\alpha}\right|\right)}{1-\Phi\left(\left|z_{\alpha}\right|\right)} \geq\left|z_{\alpha}\right|
$$

Therefore, the condition $\|\theta\|_{T} \geq \varphi\left(\left|z_{\alpha}\right|\right) / \alpha$, which has to be satisfied for investing into stocks under conditional capital at risk, is stronger than the condition $\|\theta\|_{T} \geq\left|z_{\alpha}\right|$. The latter condition is sufficient in order to include stocks into the optimal strategy using capital at risk as a risk measure. Otherwise stated, conditional capital at risk is a more conservative risk measure than capital at risk, which is consistent with its definition.

(ii) Increasing the time horizon $T$ leads to increasing the $L^{2}$ norm of the market price of risk, so that, in case (ii), the optimal strategy changes from a pure bond strategy to a mixed bond-stocks strategy. In case (i), increasing the $L^{2}$ norm $\|\theta\|_{T}$ leads to increasing the expected wealth $E\left[X^{\pi}(T)\right]$ and decreasing conditional capital at risk $\operatorname{CCaR}\left(X_{0}, \pi, T\right)$.

(iii) As was noted in the preliminary remarks, the optimal portfolio is a weighted average of Merton's portfolio and the bond, which is an illustration of the two-fund separation theorem.

We note that the solution provided in Theorem 4.2 is not an explicit analytical solution, but it is expressed in terms of the solution of the one-dimensional equation (4.11), whose solution $\varepsilon^{*}$ can be easily computed. Analytical upper and lower bounds for $\varepsilon^{*}$ are given in the following lemma, whose proof is given in the appendix.

Lemma 4.4. The unique solution $\varepsilon^{*}$ of (4.17) satisfies the inequality

$$
\left(\|\theta\|_{T}\left(1-\frac{1}{\left|z_{\alpha}\right|^{2}}\right)-\left|z_{\alpha}\right|\right)^{+} \leq \varepsilon^{*} \leq\|\theta\|_{T}-\left|z_{\alpha}\right|,
$$

where $a^{+}:=\max \{a, 0\}$. 
Remark 4.5. Note that, for $\alpha=0.05$, that is, at the $95 \%$ confidence level, $\left(1-1 /\left|z_{\alpha}\right|^{2}\right) \approx 0.63$, (4.21) approximates the result from [14], which states

$$
\left(\frac{2}{3}\|\theta\|_{T}-\left|z_{\alpha}\right|\right)^{+} \leq \varepsilon^{*} \leq\|\theta\|_{T}-\left|z_{\alpha}\right| \quad \text { for } \alpha<0.15
$$

However, at a higher confidence level, that is, $\alpha<0.05$, (4.21) gives a better approximation for $\varepsilon^{*}$, that is, a smaller interval to which $\varepsilon^{*}$ belongs.

\section{Portfolio Optimization with Respect to Conditional Capital at Risk}

We now turn to the problem of maximizing wealth subject to constrained $\mathrm{CCaR}$, that is,

$$
\max _{\pi \in Q} E\left[X^{\pi}(T)\right] \text { subject to } \operatorname{CCaR}\left(X_{0}, \pi, T\right) \leq C .
$$

Using (2.18), the above problem can be written in the form

$$
\max _{\pi \in Q} X_{0} R(T) \exp \left(\langle B, \pi\rangle_{T}\right) \quad \text { subject to } X_{0} R(T)\left(1-\exp \left(g_{\alpha}(\pi, T)\right)\right) \leq C,
$$

which is equivalent to

$$
\max _{\pi \in Q}\langle B, \pi\rangle_{T} \quad \text { subject to } \exp \left(g_{\alpha}(\pi, T)\right) \geq 1-\frac{C}{X_{0} R(T)}
$$

Since $\mathrm{CCaR}$, from its definition, is smaller than total wealth, to avoid trivial cases we only consider $C$ such that

$$
C<X_{0} R(T)
$$

Under condition (5.4), problem (5.3) can be written as

$$
\max _{\pi \in \mathcal{Q}}\langle B, \pi\rangle_{T} \quad \text { subject to } g_{\alpha}(\pi, T) \geq c,
$$

where

$$
c=\ln \left(1-\frac{C}{X_{0} R(T)}\right) .
$$

Note that condition (5.4) guarantees that $c$ is well defined. Using the dimension reduction procedure of $[2,14]$ (see Section 4), we can write problem (5.5) as a one-parameter optimization problem:

$$
\max _{\varepsilon \geq 0} \max _{\pi \in Q_{\varepsilon}}\langle B, \pi\rangle_{T} \quad \text { subject to } g_{\alpha}(\pi, T) \geq c .
$$


The solution to (5.7) is given in the following theorem.

Theorem 5.1. Suppose that the constant risk level $C$ satisfies the following condition:

$$
\begin{aligned}
X_{0} R(T)\left(1-\frac{1}{\alpha} \exp \left(\varepsilon^{*}\|\theta\|_{T}\right) \Phi\left(-\left|z_{\alpha}\right|-\varepsilon^{*}\right)\right) & \leq C<X_{0} R(T), \text { if }\|\theta\|_{T} \geq \frac{\varphi\left(\left|z_{\alpha}\right|\right)}{\alpha} \\
0 & \leq C<X_{0} R(T), \text { if }\|\theta\|_{T}<\frac{\varphi\left(\left|z_{\alpha}\right|\right)}{\alpha},
\end{aligned}
$$

where $\varepsilon^{*}$ is defined in Theorem 4.2. Then the optimal solution to problem (5.7) is

$$
\pi_{\varepsilon^{* *}}(t)=\frac{\varepsilon^{* *}}{\|\theta\|_{T}}\left(\sigma(t) \sigma(t)^{\prime}\right)^{-1} B(t)
$$

where $c$ is defined in (5.6), and $\varepsilon^{* *} \in\left[\varepsilon^{*}, \infty\right)$ is the unique solution of the equation

$$
h(\varepsilon):=\varepsilon\|\theta\|_{T}+\ln \left(\Phi\left(-\left|z_{\alpha}\right|-\varepsilon\right)\right)-\ln \alpha-c=0 .
$$

The corresponding expected wealth is equal to

$$
E\left[X^{\pi_{\varepsilon^{* *}}}(T)\right]=X_{0} R(T) \exp \left(\varepsilon^{* *}\|\theta\|_{T}\right),
$$

with the corresponding conditional capital at risk:

$$
\operatorname{CCaR}\left(\mathrm{X}_{0}, \pi_{\varepsilon^{* *}}, T\right)=\mathrm{C}
$$

The efficient frontier is given by the following curve, whose first component is an increasing function of $C$ defined implicitly through (5.6), (5.10), and (5.11):

$$
\left\{\left(C, E\left[X^{\pi_{\varepsilon}^{* *}}(T)\right]\right) \mid C \text { satisfies (5.8) }\right\} \text {. }
$$

Proof of Theorem 5.1. Proposition 4.1 states that the problem

$$
\max _{\pi \in \mathcal{Q}_{\varepsilon}}\langle B, \pi\rangle_{T}
$$

has the optimal solution

$$
\pi_{\varepsilon}(t)=\frac{\varepsilon}{\|\theta\|_{T}}\left(\sigma(t) \sigma(t)^{\prime}\right)^{-1} B(t)
$$

Substituting (5.15) into (5.7) transforms it into the problem

$$
\max _{\varepsilon \geq 0} \varepsilon\|\theta\|_{T} \quad \text { subject to } g_{\alpha}\left(\pi_{\varepsilon}, T\right) \geq c \text {. }
$$


Using (5.10), the above problem can be written as

$$
\max _{\varepsilon \geq 0} \varepsilon\|\theta\|_{T} \quad \text { subject to } h(\varepsilon)=\varepsilon\|\theta\|_{T}+\ln \left(\Phi\left(-\left|z_{\alpha}\right|-\varepsilon\right)\right)-\ln \alpha-c \geq 0
$$

Clearly, the problem achieves its optimal solution for maximal $\varepsilon$ for which the constraint is satisfied. The solution to this problem is given in the following lemma, whose proof is given in the appendix.

Lemma 5.2. Under condition (5.8), the equation $h(\varepsilon)=0$ has a maximal solution $\varepsilon^{* *} \in\left[\varepsilon^{*}, \infty\right)$. Furthermore, $h^{\prime}(\varepsilon)<0$, for $\varepsilon>\varepsilon^{*}$.

We substitute $\varepsilon^{* *}$ into (5.15) to get the optimal portfolio

$$
\pi_{\varepsilon^{* *}}(t)=\frac{\varepsilon^{* *}}{\|\theta\|_{T}}\left(\sigma(t) \sigma(t)^{\prime}\right)^{-1} B(t)
$$

which yields

$$
E\left[X^{\pi_{\varepsilon^{* *}}}(T)\right]=X_{0} R(T) \exp \left(\varepsilon^{* *}\|\theta\|_{T}\right), \quad \operatorname{CCaR}\left(X_{0}, \pi_{\varepsilon^{* *}}, T\right)=C
$$

Finally, to prove that the expected wealth is an increasing function of the risk constant $C$, we rewrite (5.10) in the form

$$
h\left(\varepsilon^{* *}\right)=\varepsilon^{* *}\|\theta\|_{T}+\ln \left(\Phi\left(-\left|z_{\alpha}\right|-\varepsilon^{* *}\right)\right)-\ln \alpha-\ln \left(1-\frac{C}{X_{0} R(T)}\right)=0,
$$

which defines $\varepsilon^{* *}$ as an implicit function of $C$. Differentiating the above, we get

$$
\frac{\partial h}{\partial \varepsilon^{* *}} \frac{d \varepsilon^{* *}}{d C}+\frac{\partial h}{\partial C}=0
$$

so that

$$
\frac{d \varepsilon^{* *}}{d C}=-\frac{\partial h / \partial C}{\partial h / \partial \varepsilon^{* *}}
$$

Since $(\partial h / \partial C)=\left(1 / X_{0} R(T)-C\right)$, and since $C<X_{0} R(T)$, it follows that $\partial h / \partial C>0$. From Lemma 5.2 it follows that $d h / d \varepsilon<0$, for all $\varepsilon>\varepsilon^{*}$, and hence for $\varepsilon^{* *}$, so that $d \varepsilon^{* *} / d C>0$. Thus, $\varepsilon^{* *}$ is an increasing function of $C$. From (5.11), we see that the expected wealth is an increasing function of $\varepsilon^{* *}$, and consequently of $C$. This completes the proof of the theorem.

Remark 5.3. If a risk-averse investor decides to take the minimal risk which, in the first case, means taking for the risk constant

$$
C=X_{0} R(T)\left(1-\frac{1}{\alpha} \exp \left(\varepsilon^{*}\|\theta\|_{T}\right) \Phi\left(-\left|z_{\alpha}\right|-\varepsilon^{*}\right)\right),
$$


implying

$$
c=-\ln \alpha+\varepsilon^{*}\|\theta\|_{T}+\ln \left(\Phi\left(-\left|z_{\alpha}\right|-\varepsilon^{*}\right)\right),
$$

then the unique solution of the equation

$$
\varepsilon\|\theta\|_{T}+\ln \left(\Phi\left(-\left|z_{\alpha}\right|-\varepsilon\right)\right)-\ln \alpha-c=0
$$

is $\varepsilon^{* *}=\varepsilon^{*}$.

In the second case, for a risk averse investor, the minimal risk constant is $C=0$, which implies $c=0$, leading to the equation

$$
h(\varepsilon)=\varepsilon\|\theta\|_{T}+\ln \left(\Phi\left(-\left|z_{\alpha}\right|-\varepsilon\right)\right)-\ln \alpha=0, \quad \text { with } h(0)=0, \quad h^{\prime}(\varepsilon) \leq 0,
$$

so that the unique solution of the equation $h(\varepsilon)=0$ is $\varepsilon^{* *}=0$.

Therefore, in both cases, choosing the minimal risk constant leads to the optimal strategies coinciding with the strategies from the previous chapter, in which minimal $\operatorname{CCaR}\left(X_{0}, \pi, T\right)$ was determined.

\section{Applications}

To illustrate the results from previous sections, we give some numerical examples. We recall that the system of SDEs that models the stocks' prices is

$$
d S_{i}(t)=S_{i}(t)\left(b_{i}(t) d t+\sum_{j=1}^{m} \sigma_{i j}(t) d W^{j}(t)\right), \quad S_{i}(0)>0, i=1, \ldots, m,
$$

and that the stocks' returns variance-covariance matrix, which we denote by $\Gamma(t)$, is equal to $\sigma(t) \sigma(t)^{\prime}$. We also recall that $\Gamma(t)$ can be decomposed as

$$
\Gamma(t)=v(t) \Delta(t) v(t),
$$

where $\Delta(t)$ is the stocks' returns correlation matrix, and $v(t)$ is a diagonal matrix with the entries equal to the stocks' returns standard deviations. Therefore, from (6.2) we get

$$
\Gamma(t)=\sigma(t) \sigma(t)^{\prime}=v(t) \Delta(t) v(t)
$$

Although, theoretically, we assume that the vector of independent Brownian motions $W(t)$ is $\left\{\mathcal{F}_{t}\right\}_{t \in[0, T]}$-adapted, that is, known at time $t \in[0, T]$, it is a common practice that we only observe $\Gamma(t)$ or, equivalently, $v(t)$ and $\Delta(t)$, but not $\sigma(t)$. From (6.3) we see that this leads to a nonunique decomposition of $\Gamma(t)$ into the product $\sigma(t) \sigma(t)^{\prime}$. Despite that fact, the Euclidean norm, and consequently, the $L^{2}$-norm of the market price of risk, is uniquely determined by

$$
\|\theta(t)\|^{2}=\left\|\sigma(t)^{-1} B(t)\right\|^{2}=B(t)^{\prime}(\sigma(t) \sigma(t))^{-1} B(t),
$$


or, in the terms of the standard deviation and the correlation matrix, as

$$
\|\theta(t)\|^{2}=B(t)^{\prime} v(t)^{-1} \Delta(t)^{-1} v(t)^{-1} B(t)
$$

To keep the exposition simple we consider the cases where the interest rate $r(t)$, as well as the volatility matrix, is constant, and the number of stocks is $m=3$. In all numerical computations and the corresponding plots we use an annual time scale for the drifts, standard deviations, and the correlation matrix $\Delta(t)$ of the 3 stocks. We model time dependency in the drift $b_{i}(t)$ by assuming that it oscillates periodically around a constant level $\mu_{i}$. In order to capture cycles in the economy or in the dynamics of the stocks we model the drifts as

$$
b_{i}(t)=\mu_{i}+\beta_{i} \cos \left(\varphi_{i} t\right), \quad i=1,2,3 .
$$

We note that the above model for stocks' drifts was already used in [17], as the amplitude and frequency coefficients $\beta_{i}$ and $\varphi_{i}$ allow a high degree of flexibility in adjusting the shape of this time dependency. We also note that, when modeling real market data, it is quite easy to deal with the above functional form and estimate these two parameters by maximum likelihood techniques, rather than detrending the data.

We now look at four special cases with the following characteristics.

(i) We let $\varphi_{1}=\varphi_{2}=\varphi_{3}=\varphi$, with $\varphi=0.75$; that is, the economic cycles of all three stocks are the same. We consider $\beta_{1}=0.75 \beta, \beta_{2}=0.5 \beta, \beta_{3}=0.25 \beta$, with $\beta=0.015$, which corresponds to a $1.5 \%$ deviation around the constant values $\mu_{i}$.

(ii) We assume that the interest rate is $r=0.05$ and numerically explore the sensitivity of the optimal strategies with respect to $\mu_{i}$ and $\rho$.

(iii) We assume that the stocks' returns have constant standard deviations given as follows:

$$
v_{1}=20 \%, \quad v_{2}=25 \%, \quad v_{3}=30 \%
$$

To emphasize the importance of the diversification effect and, consequently, of the market price of risk, for the optimal strategies, in Examples 6.2, 6.3, and 6.4 we keep the same drift and volatility coefficients and change the correlation matrix.

Example 6.1. We assume that $\mu_{1}=0.12, \mu_{2}=0.10$, and $\mu_{3}=0.08$, and that the correlation matrix is

$$
\Delta=\left[\begin{array}{ccc}
1.0 & -0.6 & -0.8 \\
-0.6 & 1.0 & 0.5 \\
-0.8 & 0.5 & 1
\end{array}\right]
$$

In Figure 2, we show (a) the stocks' drifts over a ten year period, with daily granularity, and (b) the optimal strategy, under minimal CCaR.

In this example, we see the expected result, that is, stock 1 , which has the largest constant part in the drift, and the smallest volatility is present in the optimal portfolio in 


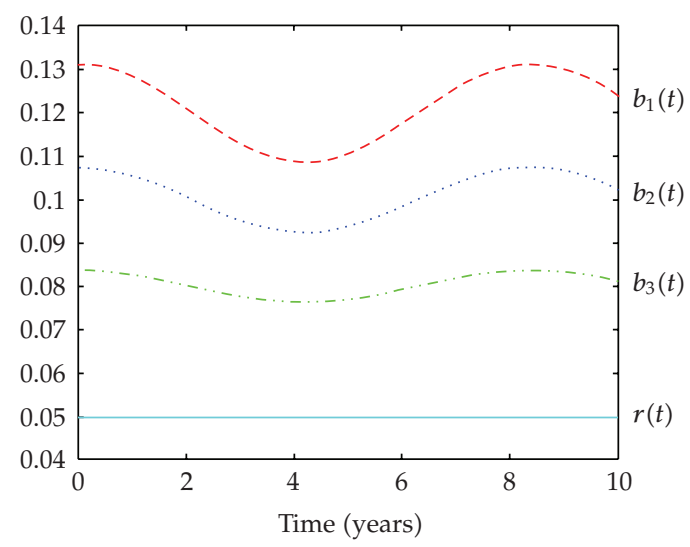

(a)

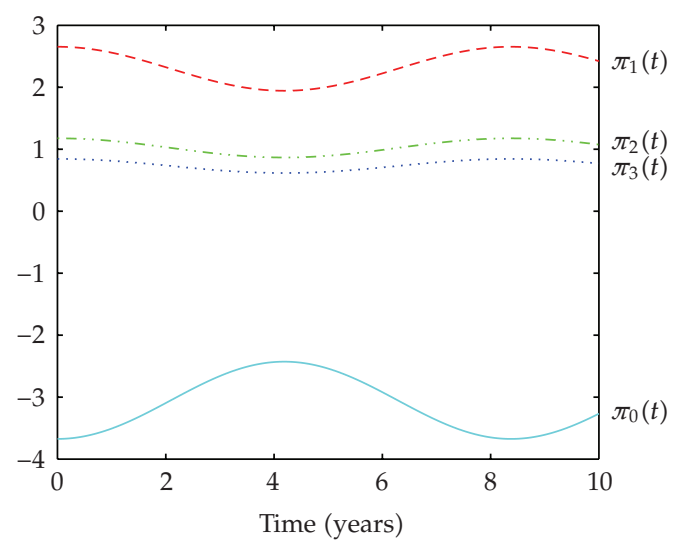

(b)

Figure 2: Plot of the Stock Drifts (a) and portfolio weights (b) for Example 6.1.

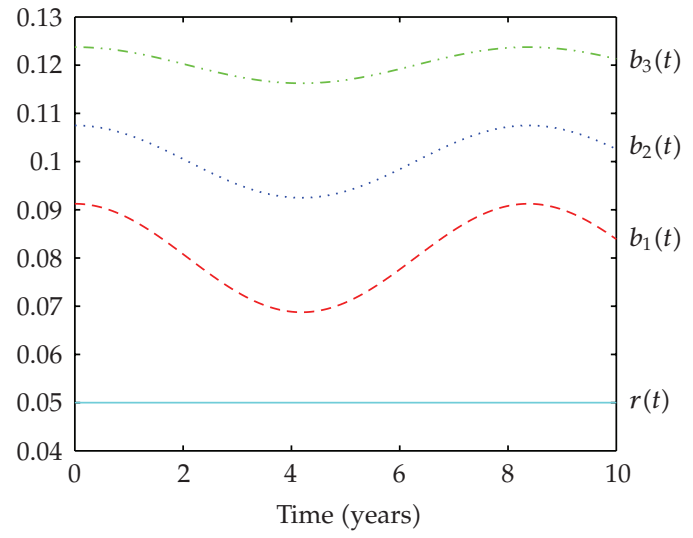

(a)

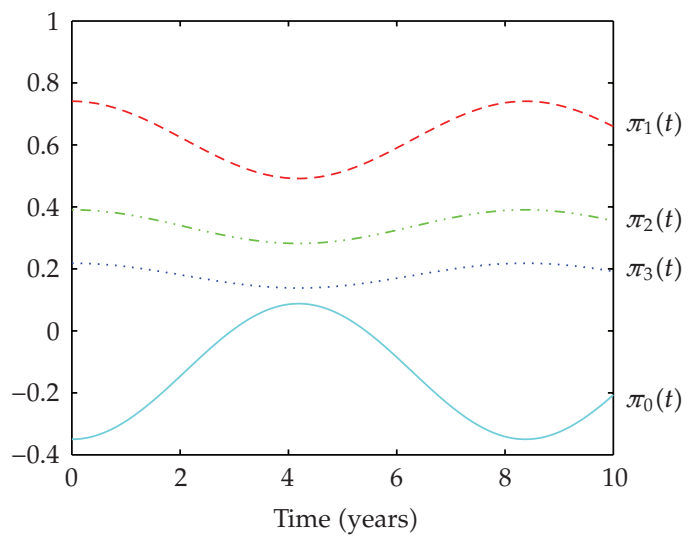

(b)

Figure 3: Plot of the Stock Drifts (a) and portfolio weights (b) for Example 6.2.

the highest percentage. However, to assume that just drifts and volatilities determine the optimal strategy would be misleading, as the following examples show.

Example 6.2. We assume that $\mu_{1}=0.08, \mu_{2}=0.10, \mu_{3}=0.12$, and that the correlation matrix is the same as in Example 6.1.

The stocks' drifts and portfolio weights are given in Figure 3.

We see that, despite the fact that stock 1 has the smallest constant part of the drift, it is still present in the optimal portfolio in the highest percentage, due to its high negative correlations with both stocks 2 and 3. 


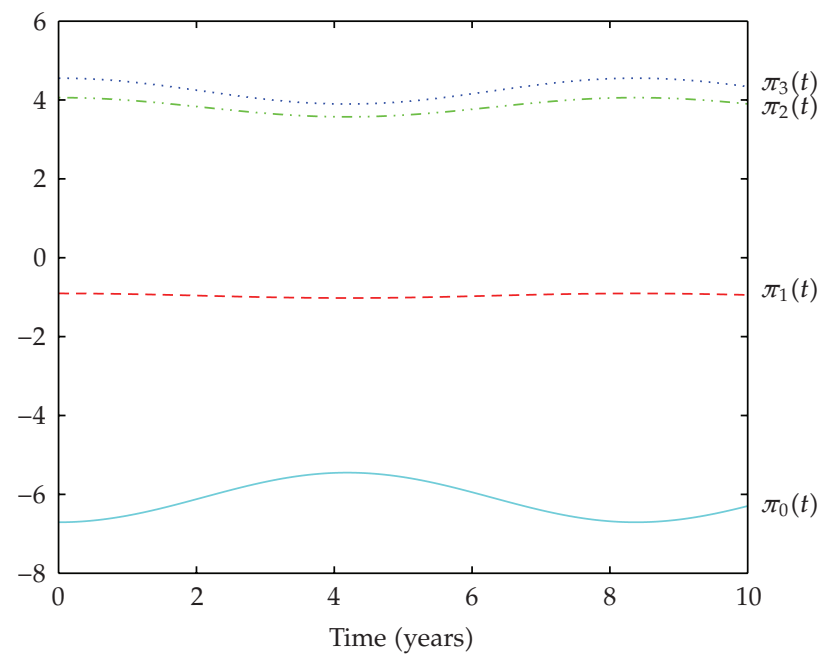

Figure 4: Portfolio Weights under Minimal CCaR for Example 6.3.

Example 6.3. In this example we assume that $\mu_{1}=0.08, \mu_{2}=0.10$, and $\mu_{3}=0.12$; that is, the stocks have the same constant parts of the drifts as in Example 6.2 but a different correlation matrix given by

$$
\Delta=\left[\begin{array}{ccc}
1.0 & -0.3 & 0.5 \\
-0.3 & 1.0 & -0.9 \\
0.5 & -0.9 & 1
\end{array}\right]
$$

The optimal strategy, under minimal CCaR, is given in Figure 4.

In this example, stocks 2 and 3, which are highly negatively correlated, are present in the optimal portfolio in percentages above $400 \%$, while stock 1 and the bond are being borrowed.

Example 6.4. In this example, we assume again that the stocks have the same constant parts of the drifts as in Examples 6.2 and 6.3, while the correlation matrix is

$$
\Delta=\left[\begin{array}{ccc}
1.0 & 0.2 & -0.3 \\
0.2 & 1.0 & 0.1 \\
-0.3 & 0.1 & 1
\end{array}\right]
$$

The criterion for investing into stocks, under the minimal CCaR, is $\|\theta\|_{T} \geq \varphi\left(\left|z_{\alpha}\right|\right) / \alpha$. For $\alpha=0.05,\left|z_{\alpha}\right|=1.645$, so that we must have $\|\theta\|_{T} \geq 2.0620$, in order to start investing into stocks. In the case of low correlation coefficients, such as in the above example, the market price of risk increases very slowly, so that it takes the time horizon of 34 years to invest into stocks with the risk level of 0.05 . For $\alpha=0.01,\left|z_{\alpha}\right|=2.33$, so that we must have $\|\theta\|_{T} \geq 2.6424$ to start investing into stocks. If we choose the risk level to be 0.01 , we have to assume the very 


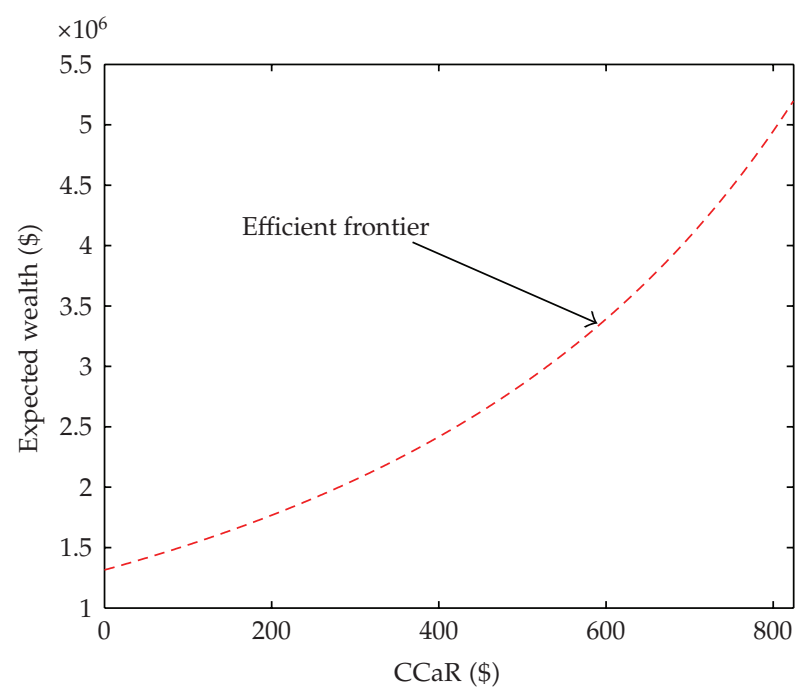

Figure 5: Efficient Frontier for Example 6.1.

Table 1

\begin{tabular}{lccccccccc}
\hline$T$ & 10 & 20 & 30 & 33 & 34 & 40 & 50 & 56 & 57 \\
\hline$\|\theta\|_{T}$ & 1.1420 & 1.5944 & 1.9344 & 2.0302 & 2.0705 & 2.2293 & 2.5030 & 2.6424 & 2.6655 \\
\hline
\end{tabular}

long time horizon, of 57 years, in order to include stocks into the optimal strategy, under minimal CCaR. The relation between $\|\theta\|_{T}$ and $T$ can be found in Table 1.

\section{Efficient Frontiers}

Figure 5 shows the efficient frontier for Example 6.1 created using Theorem 5.1. The theorem states that the expected wealth is an increasing function of $C$, which is bounded above by

$$
C_{\max }=X_{0} R(T) .
$$

In order to avoid extremely risky strategies as $C \rightarrow C_{\max }$, and for the sake of more transparency of the graphs, we restrict $C$ to the interval $\left[0,0.5 C_{\max }\right]$. We note that the efficient frontiers for the other three examples are of the same exponential form, so that we omit their graphs.

The graph given in Figure 5 illustrates the fact that the efficient frontiers are an increasing function of the risk constant $C$ which bounds CCaR.

\section{Time-CCaR-Expected Wealth Surfaces}

In Figure 6 we give a three-dimensional plot of the expected wealth as a function of time and the risk constant $C$. From Theorem 5.1 we know that the expected wealth increases when the risk constant $C$ increases. We recall that the expected wealth is also an increasing function of the time horizon $T$. The following figure illustrates these facts for Example 6.1. In order 


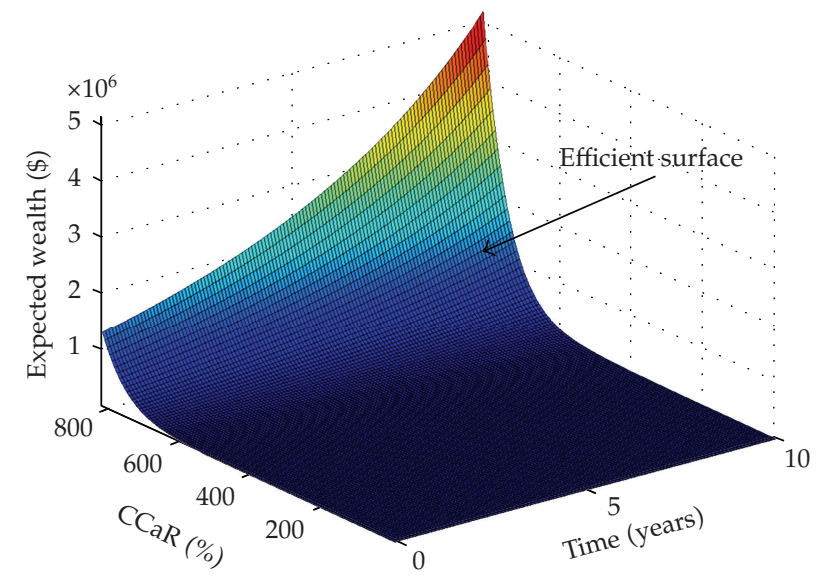

Figure 6: Time-CCaR-Expected Wealth Surface for Example 6.1.

to avoid extremely risky strategies, and to get a better representation, we restrict the upper bound for $\mathrm{CCaR}$ to the interval $\left[0,0.5 \mathrm{C}_{\max }\right]$.

\section{Conclusion}

In this work we investigated continuous time portfolio selection under the notion of conditional capital at risk, within the Black-Scholes asset pricing paradigm, with time dependent coefficients. We showed that conditional capital at risk is a coherent risk measure and proved that it satisfies an important property of strong quasiconvexity. Based on an idea from [14], generalized in [3], we introduced the fundamental dimension reduction procedure which transforms $m$-dimensional optimization problems into one-dimensional problems, within the class of admissible portfolios, which are Borel measurable, bounded, and deterministic. We further developed optimal strategies for portfolio optimization under constrained conditional capital at risk. It is important to emphasize that we considered time dependent portfolios, where the methods developed in [14] no longer work. We illustrated the two-fund separation theorem, by showing that all optimal strategies are the weighted averages of Merton's portfolio and the bond, and the weights depend on the investor's risk tolerance only. Finding optimal strategies requires solving nonlinear equations which include the cumulative distribution function of the normal random variable, so that the weights can be only found numerically. We provide several numerical examples which illustrate the importance of diversification, given by the correlation matrix. The correlation matrix significantly impacts the magnitude of the $L^{2}$ norm of the market price of risk, which, in turn, is the determining criterion for investment strategies.

\section{Appendix}

Proof of Proposition 2.5. Let

$$
\mu(t)=r(t)+B(t)^{\prime} \pi(t), \quad \eta(t)=\sigma(t)^{\prime} \pi(t) .
$$


Then the differential equation of the wealth process given by (2.5) can be written as

$$
d X^{\pi}(t)=X^{\pi}(t)(\mu(t) d t+\eta(t) d W(t)), \quad X^{\pi}(0)=X_{0} .
$$

For convenience, set $Y(t)=\ln X^{\pi}(t)$. Applying the multidimensional version of Itô's Lemma, it can be shown (see the proof of Proposition 2.1 of [3]) that $Y(t)$ follows the dynamics

$$
d Y(t)=\left(\mu(t)-\frac{1}{2}\|\eta(t)\|^{2}\right) d t+\eta(t) d W(t), \quad Y(0)=\ln \left(X_{0}\right)=Y_{0}
$$

The $\alpha$-quantile of $Y(t)$ is equal to

$$
q_{\alpha}\left(Y_{0}, \pi, t\right)=Y_{0}+\int_{0}^{t}\left(\mu(s)-\frac{1}{2}\|\eta(s)\|^{2}\right) d s-\left|z_{\alpha}\right| \sqrt{\int_{0}^{t}\|\eta(s)\|^{2} d s}
$$

so that the $\alpha$-quantile of $X(t)$ is equal to $q_{\alpha}\left(X_{0}, \pi, t\right)=\exp \left(q_{\alpha}\left(Y_{0}, \pi, t\right)\right)$. We will further simplify the notation by introducing

$$
q_{x}=q_{\alpha}\left(X_{0}, \pi, t\right), \quad q_{y}=q_{\alpha}\left(Y_{0}, \pi, t\right) .
$$

From

$$
X^{\pi}(t) I_{X^{\pi} \leq q_{x}}=\exp (Y(t)) I_{Y \leq q_{y}}
$$

where $I_{(\cdot)}$ is the corresponding indicator function, and from Bayes' theorem, we obtain

$$
\mathrm{TM}_{\alpha}\left(X^{\pi}(t)\right)=\alpha^{-1} E\left[\exp (Y(t)) I_{Y \leq q_{y}}\right]
$$

Choose $t \in[0, \mathrm{~T}]$. We will evaluate (A.7) using the characteristics of the distribution of $Y(t)$, for fixed $t$. From (A.3) we have that $Y(t)$ is a normal random variable with the parameters

$$
\mu_{y}=Y_{0}+\int_{0}^{t}\left(\mu(s)-\frac{1}{2}\|\eta(s)\|^{2}\right) d s, \quad \sigma_{y}=\sqrt{\int_{0}^{t}\|\eta(s)\|^{2} d s} .
$$

Taking into account (A.7) and (A.8), we can write

$$
\operatorname{TM}_{\alpha}\left(X^{\pi}(t)\right)=\alpha^{-1} E\left[\exp (Y(t)) I_{Y \leq q_{y}}\right]=\alpha^{-1} \int_{-\infty}^{q_{y}} \exp (y) \varphi(y) d y
$$

Using the standard integration techniques, and taking into account that $z_{\alpha}<0$, we get

$$
\operatorname{TM}_{\alpha}\left(X^{\pi}(t)\right)=\alpha^{-1} \exp \left(\mu_{y}+\frac{\sigma_{y}^{2}}{2}\right) \Phi\left(-\left|z_{\alpha}\right|-\sigma_{y}\right) .
$$


If we substitute the original notation from (A.8) and (A.1), we get (2.10). This completes the proof of Proposition 2.5.

Proof of Lemma 3.6. Let $x>0$. The function $k(x)=\ln \Phi(-x)$ is clearly decreasing. To prove that it is strictly concave, we differentiate twice to get

$$
k^{\prime \prime}(x)=\frac{\varphi(x)}{(\Phi(-x))^{2}}((1-\Phi(x)) x-\varphi(x)) .
$$

To evaluate $k^{\prime \prime}(x)$, we use the following standard inequality. For $x>0$,

$$
\frac{1}{x}-\frac{1}{x^{3}} \leq \frac{1-\Phi(x)}{\varphi(x)} \leq \frac{1}{x}
$$

Applying the above, we get that $k^{\prime \prime}(x) \leq 0$ which means that $k(x)$ is concave. To prove that $k(x)$ is strictly concave we need to show that

$$
x(1-\Phi(x))<\varphi(x) \text { for } x>0
$$

If we define the function

$$
w(x):=x(1-\Phi(x))-\varphi(x)
$$

it is an easy exercise to prove that $w(x)<0$, for all $x>0$; that is, the function $k(x)$ is strictly concave for all $x>0$, which ends the proof of Lemma 3.6.

Proof of Lemma 4.4. We prove that the optimal solution $\varepsilon^{*}$ of (4.17) satisfies (4.21). From (4.17) we have that

$$
\frac{\left(1-\Phi\left(\left|z_{\alpha}\right|+\varepsilon^{*}\right)\right)}{\varphi\left(\left|z_{\alpha}\right|+\varepsilon^{*}\right)}=\frac{1}{\|\theta\|_{T}}
$$

We use again the standard inequality (A.12). From inequality (A.12), with $x=\left|z_{\alpha}\right|+\varepsilon^{*}$, we have

$$
\frac{1}{\left|z_{\alpha}\right|+\varepsilon^{*}}-\frac{1}{\left(\left|z_{\alpha}\right|+\varepsilon^{*}\right)^{3}} \leq \frac{1}{\|\theta\|_{T}}=\frac{\left(1-\Phi\left(\left|z_{\alpha}\right|+\varepsilon^{*}\right)\right)}{\varphi\left(\left|z_{\alpha}\right|+\varepsilon^{*}\right)} \leq \frac{1}{\left|z_{\alpha}\right|+\varepsilon^{*}} .
$$

The right-hand side of the inequality implies

$$
\varepsilon^{*} \leq\|\theta\|_{T}-\left|z_{\alpha}\right| .
$$

From the left-hand side of the inequality we have

$$
\|\theta\|_{T}\left(1-\frac{1}{\left(\left|z_{\alpha}\right|+\varepsilon^{*}\right)^{2}}\right) \leq\left|z_{\alpha}\right|+\varepsilon^{*} .
$$


Since

$$
-\frac{1}{\left(\left|z_{\alpha}\right|+\varepsilon^{*}\right)^{2}} \geq-\frac{1}{\left|z_{\alpha}\right|^{2}}
$$

inequality (A.18) implies

$$
\|\theta\|_{T}\left(1-\frac{1}{\left|z_{\alpha}\right|^{2}}\right)-\left|z_{\alpha}\right| \leq \varepsilon^{*}
$$

The fact that $\varepsilon^{*} \geq 0$ and (A.20) give the desired result, so that Lemma 4.4 is proved.

Proof of Lemma 5.2. In this proof we find a maximal solution of the equation

$$
h(\varepsilon)=\varepsilon\|\theta\|_{T}+\ln \left(\Phi\left(-\left|z_{\alpha}\right|-\varepsilon\right)\right)-\ln \alpha-c=0
$$

Since $h^{\prime}(\varepsilon)=f^{\prime}(\varepsilon)$, defined by (4.15), we will apply some arguments from the proof of Theorem 4.2.

(i) Suppose that $\|\theta\|_{T} \geq \varphi\left(\left|z_{\alpha}\right|\right) / \alpha$, which means that $h^{\prime}(0)=f^{\prime}(0) \geq 0$. Then the unique maximum of $h(\varepsilon)$ is achieved at $\varepsilon^{*}$. From condition (5.8) (i) we have that

$$
\begin{aligned}
c & =\ln \left(1-\frac{C}{X_{0} R(T)}\right) \\
& \leq \ln \left(1-\left(1-\frac{1}{\alpha} \exp \left(\varepsilon^{*}\|\theta\|_{T}\right)\right) \Phi\left(-\left|z_{\alpha}\right|-\varepsilon^{*}\right)\right) \\
& =-\ln \alpha+\varepsilon^{*} \mid\|\theta\|_{T}+\ln \left(\Phi\left(-\left|z_{\alpha}\right|-\varepsilon^{*}\right)\right) .
\end{aligned}
$$

Hence

$$
\begin{aligned}
h\left(\varepsilon^{*}\right)= & \varepsilon^{*}\|\theta\|_{T}+\ln \left(\Phi\left(-\left|z_{\alpha}\right|-\varepsilon^{*}\right)\right)-\ln \alpha-c \\
\geq & \varepsilon^{*}\|\theta\|_{T}+\ln \left(\Phi\left(-\left|z_{\alpha}\right|-\varepsilon^{*}\right)\right)-\ln \alpha \\
& +\ln \alpha-\varepsilon^{*}\|\theta\|_{T}-\ln \left(\Phi\left(-\left|z_{\alpha}\right|-\varepsilon^{*}\right)\right)=0 .
\end{aligned}
$$

Therefore, $h\left(\varepsilon^{*}\right) \geq 0$. We further distinguish the following two subcases.

(a) Suppose that $C<0$. Then (A.21) implies that $h(0)=-c<0$. From the proof of Theorem 4.2, for $\varepsilon>\varepsilon^{*}, h^{\prime}(\varepsilon) \leq 0, h(\varepsilon)$ is concave, and since $h\left(\varepsilon^{*}\right) \geq 0$, it follows that the equation $h(\varepsilon)=0$ has at least one solution, with the bigger solution $\varepsilon^{* *} \in\left[\varepsilon^{*}, \infty\right)$.

(b) For $C \geq 0, h(0)=-c \geq 0$. Using the same arguments as in case (a), we get that (A.21) has a unique solution $\varepsilon^{* *} \in\left[\varepsilon^{*}, \infty\right)$. 
(ii) Suppose $\|\theta\|_{T}<\varphi\left(\left|z_{\alpha}\right|\right) / \alpha$, that is, $h^{\prime}(0)<0$. We recall that in this case $\varepsilon^{*}=0$. The definition of $h(\varepsilon)$ yields

$$
h(0)=\ln \left(\Phi\left(-\left|z_{\alpha}\right|\right)\right)-\ln \alpha-c=-c .
$$

From condition (5.8) (ii), we have that $c \leq 0$, that is, $h(0) \geq 0$. Using $h^{\prime}(\varepsilon)=f^{\prime}(\varepsilon)$, and the proof of Theorem 4.2, we have

$$
h^{\prime \prime}(\varepsilon) \leq 0 ; \quad h^{\prime}(0)=\|\theta\|_{T}-\frac{\varphi\left(\left|z_{\alpha}\right|\right)}{\alpha}<0 .
$$

Therefore, $h^{\prime}(\varepsilon)<0$ for all $\varepsilon \geq 0$, with $h(0) \geq 0$, so that $h(\varepsilon)=0$ has a unique solution $\varepsilon^{* *} \in\left[\varepsilon^{*}, \infty\right)$. This completes the proof of Lemma 5.2.

\section{Acknowledgments}

This research was partially supported by the National Science and Engineering Research Council of Canada and the Network Centre of Excellence, Mathematics of Information Technology and Complex Systems.

\section{References}

[1] P. Jorion, Value at Risk: The New Benchmark for Managing Financial Risk, McGraw-Hill, New York, NY, USA, 2nd edition, 2000.

[2] S. Emmer, C. Klüppelberg, and R. Korn, "Optimal portfolios with bounded capital at risk," Mathematical Finance, vol. 11, no. 4, pp. 365-384, 2001.

[3] G. Dmitrašinović-Vidović, A. Lari-Lavassani, X. Li, and T. Ware, Dynamic Portfolio Selection Under Capital at Risk, University of Calgary Yellow Series, University of Calgary, Alberta, Canada, 2003, Report 833.

[4] G. Dmitrašinović-Vidović, Portfolio selection under downside risk measures, Ph.D. thesis, University of Calgary, Alberta, Canada, 2004.

[5] C. Acerbi, "Spectral measures of risk: a coherent representation of subjective risk aversion," Journal of Banking and Finance, vol. 26, no. 7, pp. 1505-1518, 2002.

[6] G. Dmitrašinović-Vidović and T. Ware, "Asymptotic behaviour of mean-quantile efficient portfolios," Finance and Stochastics, vol. 10, no. 4, pp. 529-551, 2006.

[7] P. Artzner, F. Delbaen, J.-M. Eber, and D. Heath, "Coherent measures of risk," Mathematical Finance, vol. 9, no. 3, pp. 203-228, 1999.

[8] C. Acerbi and D. Tasche, "On the coherence of expected shortfall," Journal of Banking and Finance, vol. 26, no. 7, pp. 1487-1503, 2002.

[9] H. Föllmer and A. Schied, "Convex measures of risk and trading constraints," Finance and Stochastics, vol. 6, no. 4, pp. 429-447, 2002.

[10] G. Pflug, "Some remarks on the value-at-risk and the conditional value-at-risk," in Probabilistic Constrained Optimization, vol. 49 of Methodology and Applications, Kluwer Academic Publishers, Dordrecht, The Netherlands, 2000.

[11] F. Riedel, "Dynamic coherent risk measures," Stochastic Processes and Their Applications, vol. 112, no. 2, pp. 185-200, 2004.

[12] T. Rockafellar and S. Uryasev, "Conditional value at risk: optimization algorithms and applications," Financial Engineering News, vol. 14, 2000.

[13] D. Tasche and D. Bundesbank, "Expected shortfall and beyond," in Statistical Data Analysis Based on the L1-Norm and Related Methods, Statistics for Industry and Technology, Springer, Berlin, Germany, 2002. 
[14] S. Emmer, C. Klüppelberg, and R. Korn., Optimal Portfolios with Bounded Downside Risks, Working Paper, Center of Mathematical Sciences, Munich University of Technology, Munich, Germany, 2000.

[15] I. Karatzas and S. E. Shreve, Methods of Mathematical Finance, Applications of Mathematics, Springer, New York, NY, USA, 1999.

[16] M. S. Bazaraa and C. M. Shetty, Nonlinear Programming, John Wiley \& Sons, New York, NY, USA, 1979.

[17] A. Lari-Lavassani, A. A. Sadeghi, and T. Ware, Modeling and Implementing Mean Reverting Price Processes in Energy Markets, Electronic Publications of the International Energy Credit Association, 2001, http://www.ieca.net/. 


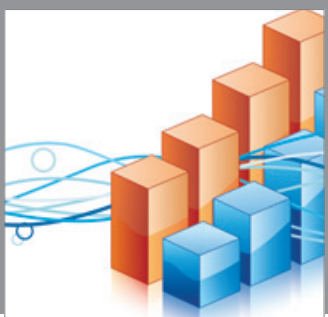

Advances in

Operations Research

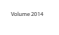

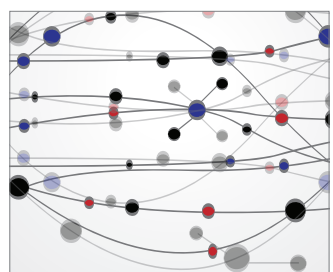

\section{The Scientific} World Journal
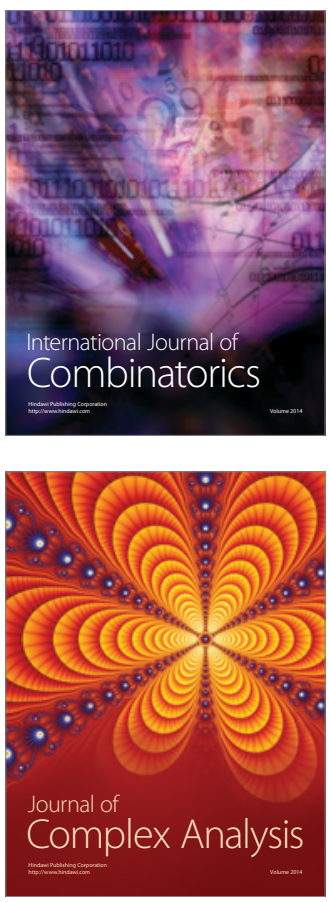

International Journal of

Mathematics and

Mathematical

Sciences
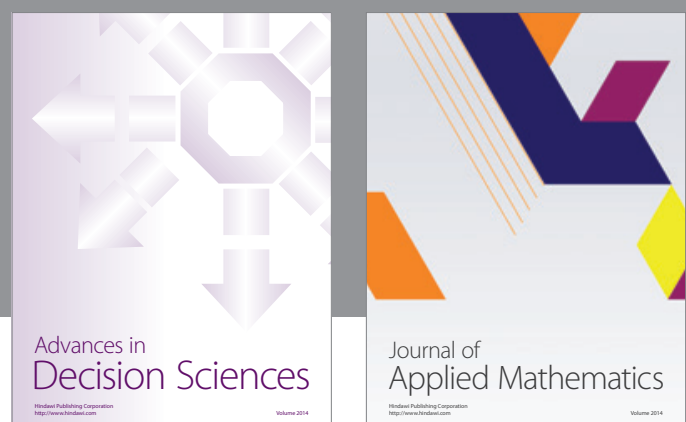

Journal of

Applied Mathematics
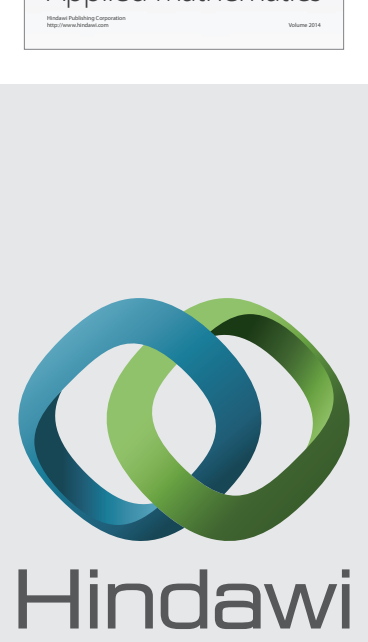

Submit your manuscripts at http://www.hindawi.com
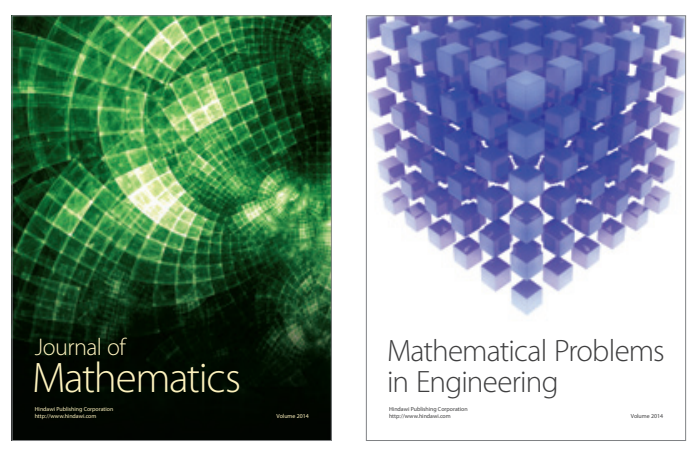

Mathematical Problems in Engineering
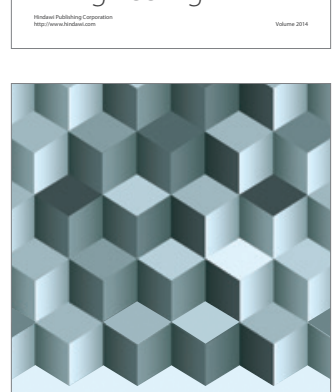

Journal of

Function Spaces
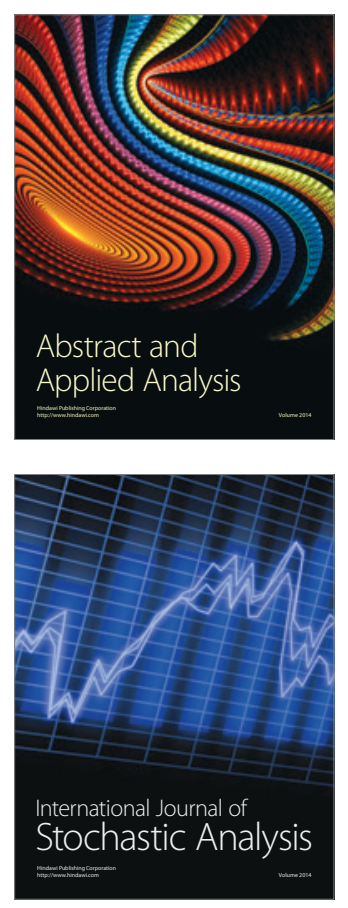

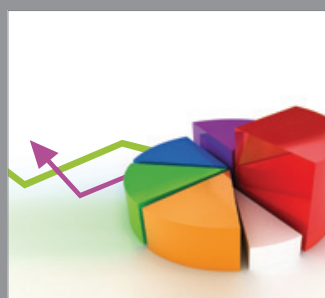

ournal of

Probability and Statistics

Promensencen
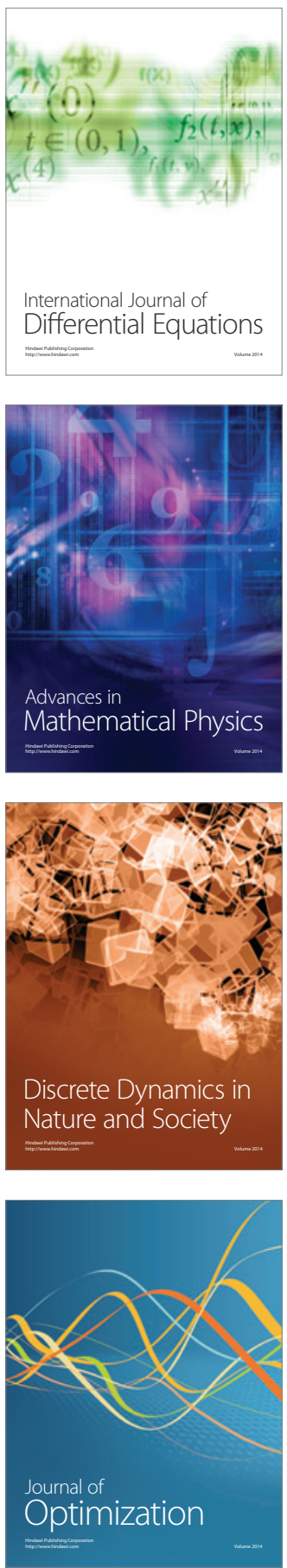